\title{
Analysis of Needlestick and Similar Injuries over 10 Years from April 2004 at Tokyo Dental College Chiba Hospital
}

\author{
Kazuki Morinaga $^{1,2)}$, Keiko Hagita ${ }^{1,3)}$, Takashi Yakushiji ${ }^{1,4)}$, Hitoshi Ohata ${ }^{1,4)}$, \\ Kenji Sueishi $^{1,5)}$ and Takashi Inoue ${ }^{1,6)}$ \\ ${ }^{1)}$ Infection Control Committee, Tokyo Dental College Chiba Hospital, \\ 1-2-2 Masago, Mihama-ku, Chiba 261-8502, Japan \\ 2) Department of Endodontics and Clinical Cariology, Tokyo Dental College, \\ 1-2-2 Masago, Mihama-ku, Chiba 261-8502, Japan \\ 3) Division of Clinical Laboratory, Tokyo Dental College, \\ 1-2-2 Masago, Mihama-ku, Chiba 261-8502, Japan \\ ${ }^{4)}$ Department of Oral and Maxillofacial Surgery, Tokyo Dental College, \\ 1-2-2 Masago, Mihama-ku, Chiba 261-8502, Japan \\ 5) Department of Orthodontics, Tokyo Dental College, \\ 1-2-2 Masago, Mihama-ku, Chiba 261-8502, Japan \\ ${ }^{6)}$ Department of Clinical Pathophysiology, Tokyo Dental College, \\ 1-2-2 Masago, Mihama-ku, Chiba 261-8502, Japan
}

Received 17 November, 2015/Accepted for publication 10 May, 2016

\begin{abstract}
Here we investigated needlestick and similar injuries reported over a 10-year period between April 2004 and March 2014. The purpose of this study was to prevent recurrence and reduce the incidence of such injuries at Tokyo Dental College Chiba Hospital. The Division of Medical Risk Management at Chiba Hospital anonymized the data to protect personal information prior to analysis. A total of 213 injuries occurred over the 10-year period investigated, but the number of cases decreased yearly. Many cases involved dental undergraduate students and dentists, followed by trainee dentists, students at the school of dental hygiene, nurses, dental hygienists, and cleaners. Suture needles, followed by injection needles, were the top two most common injury-causing instruments, contributing to approximately $50 \%$ of the total number of such cases. Many injection needle injuries occurred during tidying up, while those caused by suture needles occurred during dental treatment. Taken together, these findings suggest the importance of strict adherence to guidelines provided in safety manuals on error-free procedures and handling of instruments. Improvement in the ability to sense potential risk is essential if such injuries are to be avoided.
\end{abstract}

Key words: Needlestick injury - Occupational infection Infection control team — Medical safety 


\section{Introduction}

Health care professionals are at constant risk of exposure to bodily fluids. One characteristic of dental treatment is that it is not only surgically invasive procedures that pose the risk of exposure to fluids such as saliva and blood: this is true also of all other procedures, including those involved in general endodontics and periodontics. Furthermore, the technical specificity of the instruments used to provide such treatment requires specific measures to be implemented if this is to be avoided.

The Infection Control Committee and Infection Control Team at Tokyo Dental College Chiba Hospital have produced an infection control manual, organized lectures for dental college students, and held training workshops for staff, all aimed at heightening awareness of the need to adhere to standard precautions in preventing needlestick and other similar injuries. Such accidents continue to occur, however, albeit with decreasing frequency.

Other factors also have a bearing on this issue. For example, it was reported that many patients tend not to report past medical history when receiving dental treatment ${ }^{7)}$; and patients may not even be aware that they have an infection at all. Given this situation, it is evident that measures against occupational infections due to needlestick or similar injuries are extremely important in maintaining staff health and educating students. This indicates the need to assess the occurrence of such injuries and what measures are being taken to prevent them.

The fact that a large number of such injuries occur in students is a major issue that needs be addressed, even though this could be viewed as solely a university hospital-specific problem. Indeed, needlestick and similar injuries at medical ${ }^{4,89}$ and dental educational institutions have been discussed in Japan ${ }^{1-3,12)}$ and other countries ${ }^{5,6,10,11)}$, including England, Germany, and Australia.

The purpose of this study was to investigate reports of needlestick and similar injuries to ascertain their current status. It is hoped that the results will help establish more effective measures to prevent the recurrence and reduce the incidence of such injuries.

\section{Methods}

Data contained in needlestick and similar injury reports filed over a 10-year period between April 2004 and March 2014 were anonymized by the Division of Medical Risk Management at Tokyo Dental College Chiba Hospital. The aggregated data were then analyzed according to profession.

Items addressed comprised number of cases, month in which injury occurred, time of occurrence, proportion of professionals and workers affected, and injury-causing instrument.

\section{Results}

\section{Number of cases}

A total of 213 needlestick and similar injuries occurred over the 10-year period investigated (Fig. 1). However, the number of patients visiting our hospital annually has changed markedly: for example, it was 233,000 in 2004 and 279,000 in 2012, an increase of approximately 46,000 . The number of infections per 10,000 outpatients is shown in Fig. 2. The largest number, 1.59, was observed in 2006, but this then dropped to 0.36 in 2012 , representing only one fifth of the peak level.

\section{Month in which injury occurred}

The number of needlestick and similar injuries was particularly high in June (Fig. 3).

\section{Time of occurrence}

The number of needlestick and similar injuries was high between 10:00 and 11:00, and between 15:00 and 16:00 and 16:00 and 17:00 (Fig. 4).

\section{Injury-causing instrument}

Suture needles followed by injection nee- 


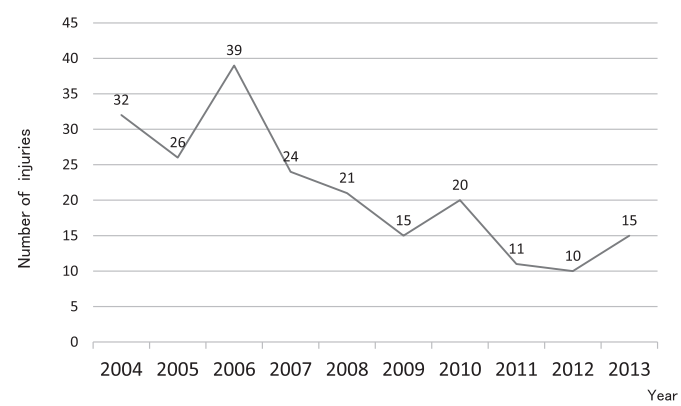

Fig. 1 Number of needlestick and similar injuries by year

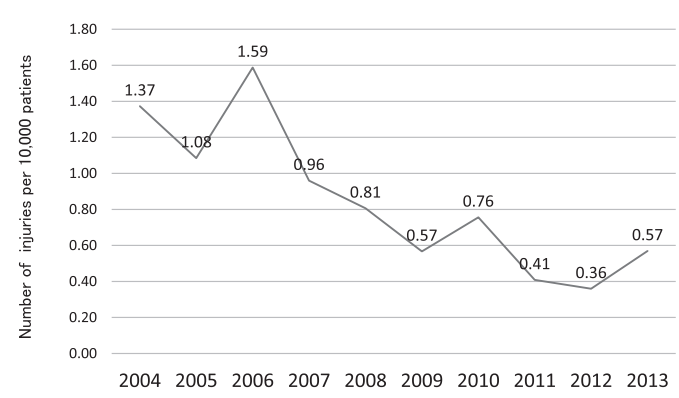

Fig. 2 Number of needlestick and similar injuries per 10,000 patients

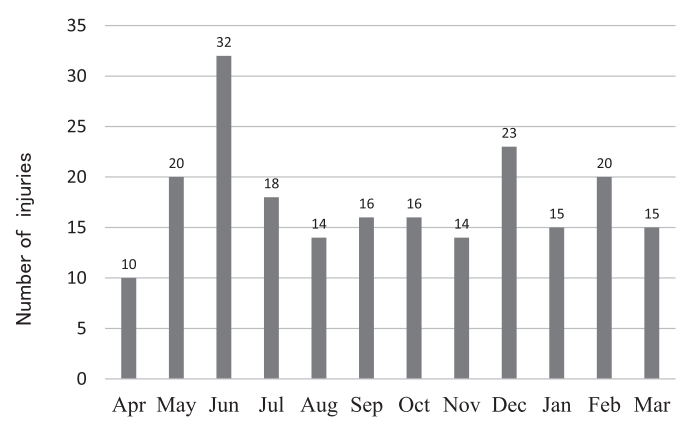

Fig. 3 Month of occurrence

dles were the two most common instruments causing injuries. Cases involving these needles accounted for approximately half of the total number of such cases (Fig. 5). Other injurycausing instruments were (in the order of the highest to the lowest): explorers, rotary cutting instruments, periodontal scalers, and

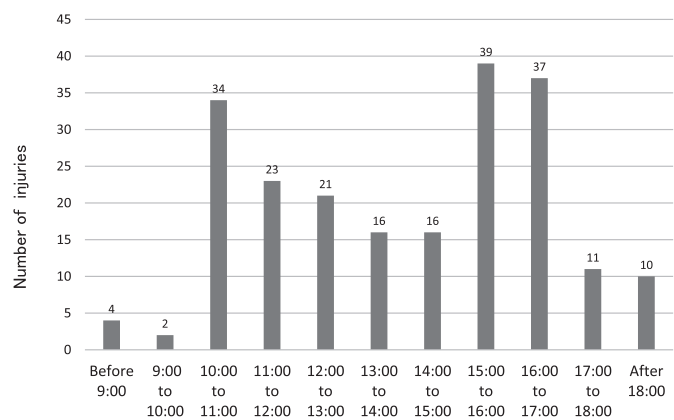

Fig. 4 Time of occurrence

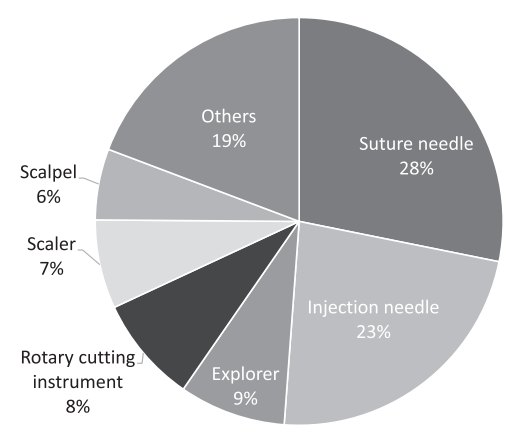

Fig. 5 Injury-causing instrument

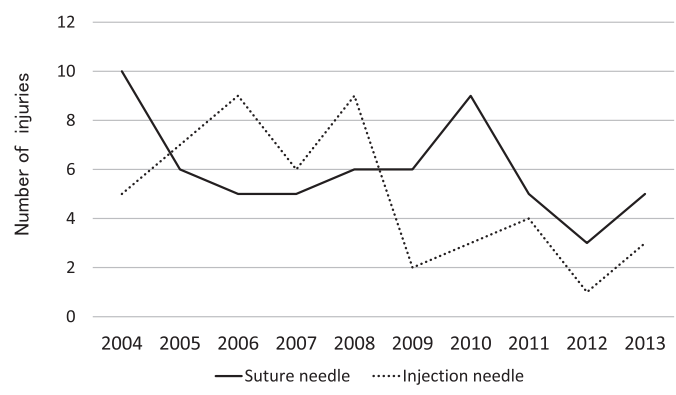

Fig. 6 Shift in pattern of needlestick and similar injuries caused by suture and injection needles by year

scalpels. Instruments used in endodontic treatment (e.g., reamers and Gates-Glidden drills), impression net trays, carving knives, and design knives also caused injuries, but only rarely. Shifts in the pattern of injuries caused by suture and injection needles, which accounted for approximately $50 \%$ of all such 


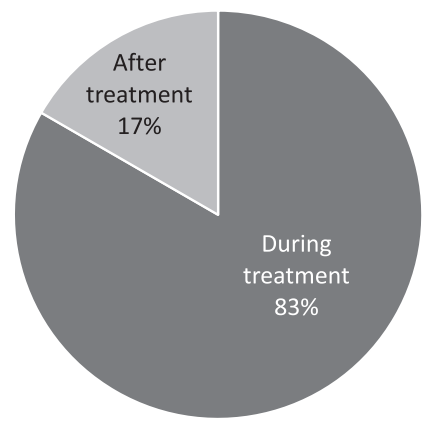

Suture needle

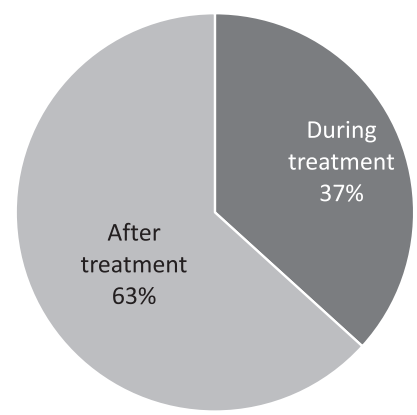

Injection needle

Fig. 7 Situation at time of injury

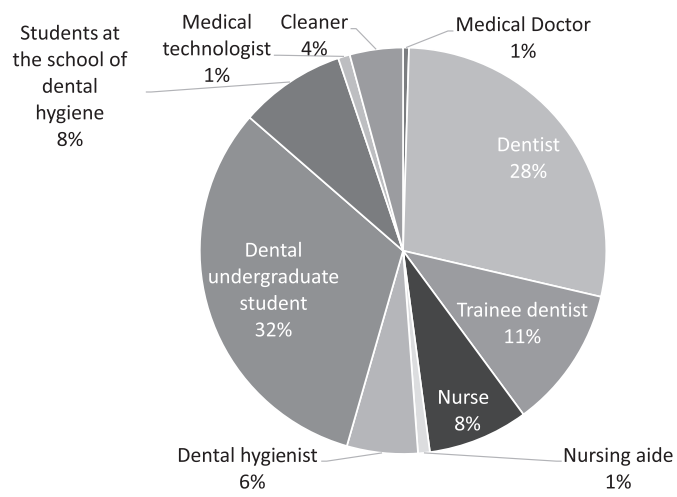

Fig. 8 Proportion of professions in injury cases

cases, are shown in Fig. 6. It was clear that although there was an overall reduction in the number of injuries, the reduction in the number of injuries due to suture needles was not so great.

The situation at the time of injury caused by suture and injection needles is shown in Fig. 7. Most injuries by suture needles occurred during dental treatment, while two thirds of injuries by injection needles occurred during clearing up. Injuries from other instruments occurred mainly during dental treatment.

\section{Proportion of professionals and workers affected}

Needlestick and similar injuries in dental undergraduate students accounted for

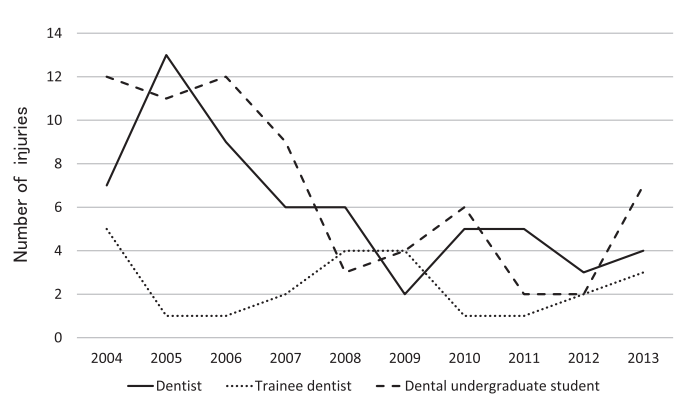

Fig. 9 Shifts in pattern of needlestick and similar injuries in dentists, trainee dentists, and dental undergraduate students by year

approximately $32 \%$ of all such cases, and those in dentists accounted for another $28 \%$. The number of cases was third highest among trainee dentists, followed by students at the school of dental hygiene, nurses, dental hygienists, and cleaners (Fig. 8). Shifts in the pattern of such cases among the top three injury-prone professions (dentists, dental undergraduate students, and trainee dentists) over the period investigated are shown in Fig. 9.

\section{Dental undergraduate students}

Lastly, we extracted data associated with dental undergraduate students and investigated the case number at each department (Fig. 10). The case number was highest in the oral surgery department, followed by the con- 


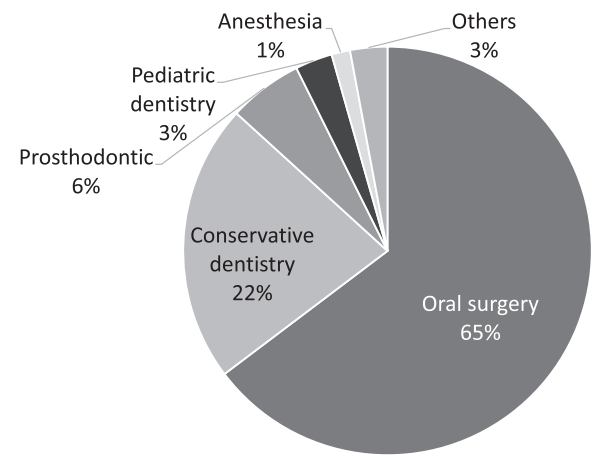

Fig. 10 Needlestick and similar injuries in dental undergraduate students by department

servative dentistry department: injuries in these two departments accounted for $87 \%$ of the total number of such cases. Also, the percentage of injuries by suture needles and injection needles among all cases was higher in dental undergraduate students than in any other group (Fig. 11). Some of these injuries occurred because the individual involved failed to implement the preventive measures recommended in the safety manual.

\section{Discussion}

The number of needlestick and similar injuries was highest in 2006, although this decreased by one fifth in 2012. It is likely that increased awareness via monthly workshops for staff and restriction of injection needle handling by undergraduate students contributed to this reduction. On the other hand, around 10 cases of such injuries still occur annually, and carelessness while providing dental treatment and ignorance of the manual have been reported.

The number of injuries was particularly high in June. Fresh undergraduate students and trainee dentists commence clinical practice in April. It is possible that growing familiarity with the environment by around June may lead to a lapse in concentration with a subsequent increase in the risk of such injury.

Many injuries occurred during the time windows of 10:00 to $11: 00,15: 00$ to $16: 00$, and

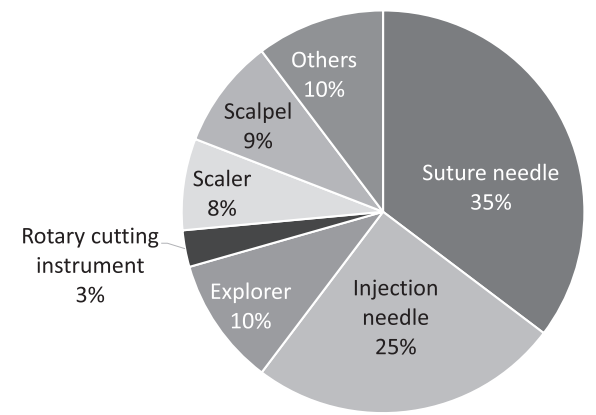

Fig. 11 Instruments causing injuries in dental undergraduate students

16:00 to 17:00, suggesting that the occurrence of injuries coincides with the peak time of outpatient visits and clearing up.

Approximately half of all injuries were caused by suture and injection needles, and the handling of these needles was associated with a high risk of injury. In order to raise risk awareness, the college has created manuals providing guidance on the handling of injection needles. This advice includes needle recapping when absolutely necessary, and also covers suture needle handing upon suture attachment. This has resulted in a tendency toward a decrease in the number of injection needle injuries (Fig. 8). Because injuries caused by injection needles tend to happen during clearing up, undergraduate students have generally been excluded from cleaning duties. On the other hand, the number of injuries caused by suture needles remains unchanged. Suture attachment is known to be associated with increased likelihood of injury, and purchasing needles with pre-attached sutures was considered at one point. This idea was abandoned, however, due to the high cost that this would involve.

Although a considerable percentage of injuries were found to occur in dental undergraduate students and dentists, this may simply reflect the fact that our hospital hosts a large number of people in those occupations. However, while the number of injuries in dentists and dental undergraduate students showed a clear reduction after the peak in 2005 and 
2006, the number of injuries in trainee dentists has remained largely unchanged. This suggests that improved awareness in dentists in general has resulted in better adherence to the guidelines on needle use and provision of more careful and thorough instructions to dental undergraduate students. On the other hand, it appears that trainee dentists are not yet able to afford time to pay sufficient attention to risks in clinical practice.

Among dental undergraduate students, the number of injuries was highest in the oral surgery and conservative dentistry departments, which is reflective of that fact that the use of sharp instruments such as suture and injection needles as part of periodontal surgery and surgical endodontics is high in these areas. The undergraduate students are renewed each year, which means that strict adherence to the recommended clinical procedures needs to be re-emphasized with each new intake.

\section{Conclusions}

Needlestick and similar injuries reported between April 2004 and March 2014 were investigated and analyzed. The following findings and conclusions were reached:

1. The total number of cases was 213. The number of cases per 10,000 outpatients in 2006 was 1.59 , but this decreased by one fifth to 0.36 in 2012.

2. Many injuries occurred in June, and the case number was highest between 15:00 and 16:00, followed by 16:00 and 17:00 and 10:00 and 11:00.

3. The percentage of injuries caused by suture needles (28\%) was the highest, followed by injection needles $(23 \%)$. Injuries caused by these two types of needle accounted for approximate half of all such cases. Other injury-causing instruments included explorers $(9 \%)$, cutting instruments $(8 \%)$, periodontal scalers $(7 \%)$, and scalpels $(6 \%)$.

4. The number of cases was particularly high among dental undergraduate students $(32 \%)$ and dentists $(28 \%)$, followed by trainee dentists $(11 \%)$, students at the school of dental hygiene $(8 \%)$, nurses $(8 \%)$, dental hygienists $(6 \%)$, and cleaners $(4 \%)$.

5 . Needlestick injuries caused by injection needles commonly occurred during clearing up $(63 \%)$, while those caused by suture needles occurred mainly during dental treatment $(83 \%)$. Injuries caused by other instruments occurred during dental treatment in general.

6. Many injuries in undergraduate students occurred at the oral surgery and conservative dentistry departments. Approximately $60 \%$ of injuries were caused by suture and injection needles.

Taken together, these results indicate the importance of strictly adhering to the advice given in manuals describing error-free procedures and handling. Furthermore, many undergraduate students sustained needlestick and similar injuries. This suggests that the introduction of workplace hazard and hazard prediction training should be considered as one possible preventive measure. Improving the ability to sense potential danger through such training and managing the physical and mental aspects of dental work is essential in preventing needlestick and similar injuries, as well as preventing malpractice in general.

This study was approved by the Ethics Committee of Tokyo Dental College (Approval no. 639).

\section{References}

1) Ibaraki Y, Ikeo M, Kawakami T, Haraguchi K, Odachi T, Matsuda T, Matsuda K (2000) Infection accident and the prevention measure in the hospital affiliated to Health Sciences University of Hokkaido. Nihon Shika Iryou Kanri Gakkai Zasshi 34:211-215. (in Japanese)

2) Isoda M, Miyatake Y, Nejima J (2006) Needlestick accidents and other sharps injuries in Tsurumi University Dental School Hospital. Tsurumi Shigaku 32:99-107. (in Japanese)

3) Kobayashi S, Ando F, Kita D, Otsu M, Ishigaki Y, Kobayashi S, Hamura A, Mishiro F, Uchikawa 
Y (2014) Assessment of needlestick injuries in the Nippon Dental University Hospital -A medical safety report for the past decadeNihon Shika Iryou Kanri Gakkai Zasshi 49: 173-180. (in Japanese)

4) Lauer AC, Reddemann A, Meier-Wronski CP, Bias H, Gödecke K, Arendt M, Peters H, Gross M (2014) Needlestick and sharps injuries among medical undergraduate students. Am J Infect Control 42: 235-239.

5) McCarthy GM, Britton JE (2000) A survey of final-year dental, medical and nursing students: occupational injuries and infection control. J Can Dent Assoc 66:561-565.

6) McDonald RI, Walsh LJ, Savage NW (1997) Analysis of workplace injuries in a dental school environment. Aust Dent J 42: 109-113.

7) Nagao Y, Kawaguchi T, Ide T, Sata M (2008) HCV or HBV infection self-disclosure to dentists. Kansenshogaku Zasshi 82:213-219. (in Japanese)

8) Patterson JM, Novak CB, Mackinnon SE, Ellis RA (2003) Needlestick injuries among medical students. Am J Infect Control 31:226-230.

9) Schmd K, Schwaqer C, Drexler H (2007) Needlestick injuries and other occupational exposures to body fluids amongst employees and medical students of a German university: incidence and follow-up. J Hosp Infect 65: 124-130.

10) Stewardson DA, Palenik CJ, McHugh ES, Burke FJT (2002) Occupational exposures occurring in students in a UK dental school. Eur J Dent Educ 6:104-113.

11) Wicker S, Rabenau HF (2010) Occupational exposures to bloodborne viruses among German dental professionals and students in a clinical setting. Int Arch Occup Environ Health 83:77-83.

12) Yoneda M, Izumi T, Suzuki N, Naito T, Yamada K, Okuda I, Iwamoto T, Masuo Y, Kojima H, Anan H, Hirofuji T (2009) Analysis of needlestick injuries in Fukuoka Dental College Medical and Dental Hospital. Nippon Shika Hozongaku Zasshi 52:168-175. (in Japanese)

\section{Correspondence:}

Dr. Kazuki Morinaga

Department of Endodontics and Clinical Cariology,

Tokyo Dental College, 1-2-2 Masago, Mihama-ku, Chiba 261-8502, Japan

E-mail: morinaga@tdc.ac.jp 